\title{
Editorial
}

Pathobiology

Pathobiology 2011;78:59-60

DOI: $\underline{10.1159 / 000323781}$

\section{Changing Pathology with Changing Drugs}

This issue is devoted to the changing role of pathology in the context of cancer. Traditionally, the contribution of pathology was primarily centered on the diagnosis and prognosis of the lesion. This was based on careful histological examination of the cellular and tissular features as well as on the boundary of the neoplastic lesion with surrounding unaffected tissues and the extent of inflammation within and around the cancerous lesion. Although new malignancy criteria, based on genetic alterations, abnormal gene expression patterns, and proteomic and especially phosphoproteomic characterization of signaling proteins in the neoplastic cell, are now available to refine (and sometimes complicate) the diagnosis and prognosis, the traditional histopathological analysis remains essential for a reliable evaluation of cancer and a comprehensive assessment of cancer process. In the review by Leong and Zhuang [this issue, pp. 45-60], this essential 'mapping' effort by the pathologist is very convincingly described in the fast-evolving context of breast cancer.

With the introduction of small-molecular-weight inhibitors of tyrosine kinases in the treatment of CML [1], efforts to identify inhibitable, hyperactive tyrosine kinases has led to very therapeutically fruitful advances in oncology [2]. The review by Bodo and Hsi [this issue, pp. 61-67] discusses how in situ staining of tyrosine-phosphorylated oncoproteins (and the standardization of such analyses) could be achieved to confirm the prognosis of hematological malignancies and suggest more targeted therapies. The review by Karpova et al. [this issue, pp. 7-21] describes and discusses the many ways in which

\section{KARGER}

Fax +4161306 1234

E-Mail karger@karger.ch

www.karger.com (c) 2011 S. Karger AG, Basel

1015-2008/11/0782-0059\$38.00/0

Accessible online at:

www.karger.com/pat tyrosine kinases (and other signaling molecules) are presently targeted to treat a variety of skin cancers. Targeting receptor tyrosine kinases with antibodies or inhibitors, as well as other signaling molecules, has also become a common therapeutic choice in the treatment of frequent epithelial neoplasms such as those of the gastrointestinal system and the kidney. How pathologists may come to suggest targeted therapies in the context of the genetic alterations and gene expression signatures they detect is discussed by Cervera and Fléjou [this issue, pp. 22-35] for tumors of the gastrointestinal tract and by Allory et al. [this issue, pp. 36-44] for tumors of the kidney. Nevertheless, targeted therapies have some limitations. Chief among these is the potential for cells to develop resistance to them (imatinib, CML). It is for this reason that targeted therapies may work best in combination either with other targeted therapies or with more traditional therapies. Targeted cancer therapies are being studied for use alone, in combination with other targeted therapies, and in combination with other cancer treatments, such as chemotherapy. The analysis of tissues under combined therapy will be a challenge as different changes will intermingle. The second challenge will be the analysis of follow-up biopsies of the same tumor or of secondary tumors after such treatments. Still, the tools for such fine analyses will have to be developed and the understanding of the results of combined therapies will require careful evaluation. Nevertheless, the ways of analyzing tissues will change as will the understanding of the underlying diseases. 
The incorporation of new genomic and proteomic analytical technologies into the pathologist's toolkit provides him or her with new and powerful possibilities to assess the proliferative status of cancer cells. With the realization that neoplastic lesions within a given histopathological type are likely to be heterogeneous, identification of hyperactive kinases or oncogenic addiction patterns provides not only a basis for subcategories with different prognosis but also, most important from the patient's point of view, more realistic molecular targets for therapy.

In the future, the pathologist is therefore likely to assume a much more active role in the management of cancer cases, moving from the position of an observer and describer to that of someone who can suggest modalities of a personalized therapy based on the functional properties of the cancer cells under scrutiny. The predominance of proliferative signatures in the most aggressive breast cancer subtypes, for instance, will certainly widen the spectrum of inhibitory interventions in selected oncogenes, both preventively and as curative therapies.

The next step will be the tissular analysis during or after therapy. Likewise, significant changes are expected in the workup of secondary versus primary tumors. The flow of information from the clinical management to the diagnosing pathologist will have to be as closely coordinated as possible as the modifications that may appear will only be understood through an exhaustive exchange of information.

Prof. Bettina Borisch and Prof. Daniel Hoessli, Geneva

References

1 Druker BJ: Translation of the Philadelphia chromosome into therapy for CML. Blood 2008;112:4808-4817.

2 Krause DS, van Etten RA: Tyrosine kinases as targets for cancer therapy. N Engl J Med 2005;35:172-187. 